\title{
Structure and morphology of perylene films grown on different substrates
}

M. Beigmohamadi, P. Niyamakom, A. Farahzadi, C. Effertz, S. Kremers, D. Brueggemann, and M. Wuttig

Citation: Journal of Applied Physics 104, 013505 (2008);

View online: https://doi.org/10.1063/1.2951900

View Table of Contents: http://aip.scitation.org/toc/jap/104/1

Published by the American Institute of Physics

\section{Articles you may be interested in}

WSXM: A software for scanning probe microscopy and a tool for nanotechnology

Review of Scientific Instruments 78, 013705 (2007); 10.1063/1.2432410

Investigation of intermolecular interactions in perylene films on $\mathrm{Au}(111)$ by infrared spectroscopy

The Journal of Chemical Physics 136, 054503 (2012); 10.1063/1.3681164

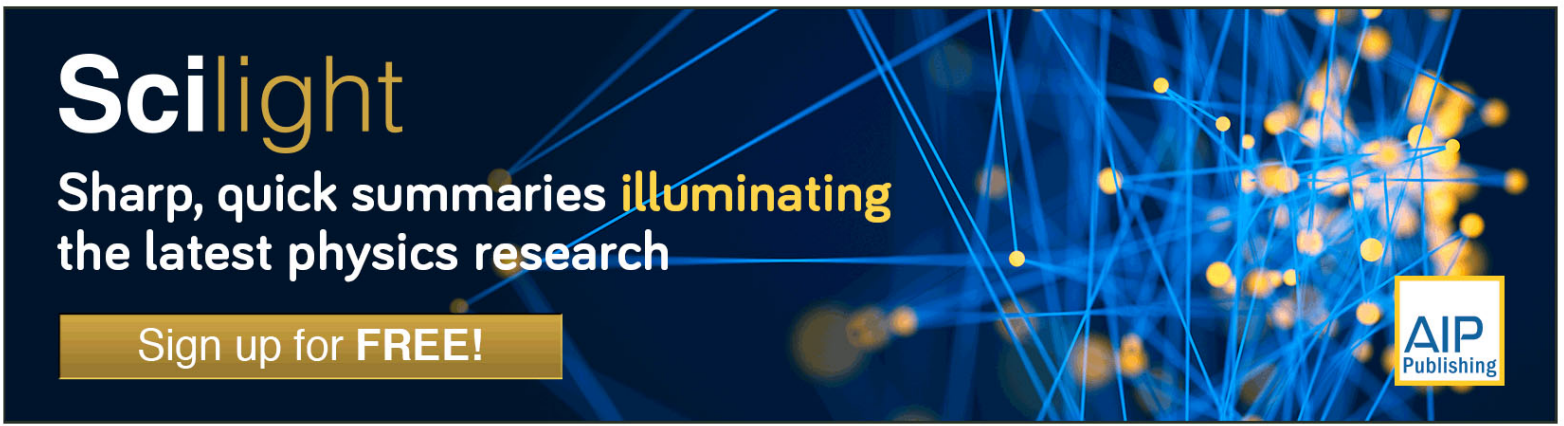




\title{
Structure and morphology of perylene films grown on different substrates
}

\author{
M. Beigmohamadi, ${ }^{\text {a) }}$ P. Niyamakom, A. Farahzadi, C. Effertz, S. Kremers, \\ D. Brueggemann, ${ }^{\text {b) }}$ and M. Wuttig ${ }^{\mathrm{c})}$ \\ Institute of Physics (IA), RWTH Aachen University, 52056 Aachen, Germany
}

(Received 8 February 2008; accepted 28 April 2008; published online 2 July 2008)

\begin{abstract}
In this study the structural and morphological properties of perylene films deposited on different substrates have been investigated. The film properties have been studied by employing a range of techniques including atomic force microscopy, x-ray diffraction, and drop shape analysis for the determination of the surface free energy. For films deposited on top of $\mathrm{Al}_{2} \mathrm{O}_{3}$, the perylene molecules have a fiber texture ordering perpendicular to the substrate, while for films deposited on top of a $\mathrm{Au}(111)$ layer, only for thicker films above $400 \mathrm{~nm}$ were diffraction peaks observed. The different growths of perylene on top of these substrates are attributed to the different strengths of the molecule-substrate interaction. (C) 2008 American Institute of Physics. [DOI: 10.1063/1.2951900]
\end{abstract}

\section{INTRODUCTION}

Over the past two decades, organic semiconductors have emerged as a technologically important class of electronic materials. ${ }^{1}$ The initial demonstration of organic field-effect transistors $^{2}$ (OFETs) has attracted the interest of industry and opened the way to practical applications for organic semiconductors. A severe limitation in the use of organic materials as semiconductors in OFET devices is the low conductivity which has been attributed to the low mobility of the charge carriers.

The electrical properties of these crystalline organic films crucially depend on their long range order. ${ }^{3}$ Therefore it has been suggested that improving the internal ordering of the organic thin film can enhance field-effect carrier mobilities. ${ }^{4}$ Since the structure of the functional organic layer and the charge injection from metallic contacts are both important in device performance, a thorough understanding of the structure and morphology of organic films in metalorganic interfaces is essential for applications in future devices.

It has been found that, in particular, the grain size strongly influences the electrical conductivity, as the overall electrical mobility of the thin film increases with grain size. ${ }^{5}$ Hence, by improving the grain size, one can optimize the electrical properties of the organic film. The possibility that the grain size may limit the mobility has motivated experimental studies of the growth morphology. It has been attempted to increase the grain size by modification of the molecule-substrate interaction. ${ }^{6}$ Furthermore it is possible to change the average grain size by tailoring the growth parameters such as the deposition rate or film thickness. ${ }^{7}$

Planar aromatic hydrocarbons (PAHs) are very promising materials for device fabrication. This group of materials has a broad intermolecular interaction potential dominated by van der Waals interactions. Thus, the molecule-substrate

\footnotetext{
a)Electronic mail: mohamadi@physik.rwth-aachen.de.

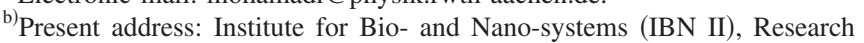
Center Jülich, 52425 Jülich, Germany.

${ }^{c)}$ Author to whom correspondence should be addressed. Electronic mail: wuttig@physik.rwth-aachen.de.
}

interaction can play a significant role in the determination of the subsequent molecule ordering and crystalline structure. While pentacene is the most frequently applied PAH, perylene is considered here which can also serve as a prototype for polycyclic aromatic hydrocarbons due to its relatively regular molecular shape. Shown in Fig. 1(a) is a schematic representation of a single perylene molecule. To form the bulk phase of $\alpha$-perylene, four molecules are arranged in one cell, where they are stand upright and adopt a face-onedge herringbone arrangement as displayed in Fig. 1(b).

At this point in time, there is still lack of systematic studies on the morphology and the structure of thin perylene films. So far the growth of perylene on top of an oxide layer has been investigated rarely. ${ }^{7,8}$ The majority of studies have focused on the growth of perylene on top of a metallic layer and mainly concentrated on the initial stage of growth. ${ }^{9,10}$ In these studies only the growth of the first few monolayers has been investigated and it is not clear whether the observed structures are observed for thicker films as well. In the case of di-indenoperylene (DIP), there exists a comparative study of the growth of DIP on top of oxide and metallic substrates. ${ }^{11}$ This study has revealed that when organic molecules are deposited on top of a metallic surface, the molecule-substrate interaction is quite different from the interaction with an oxide layer. This can lead to a different film morphology and crystalline structure. In this paper we present a detailed investigation of the structural and morpho-

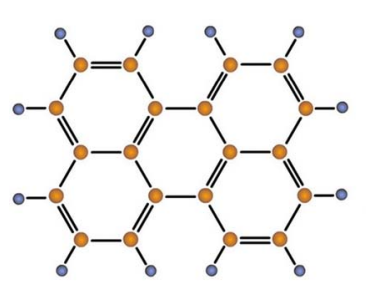

(a)

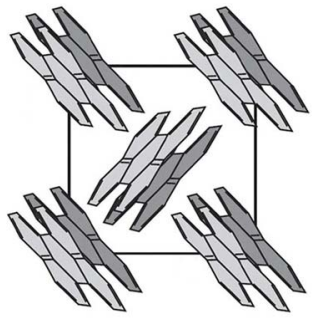

(b)
FIG. 1. (Color online) (a) Molecular structure of perylene together with (b) its crystal structure $\alpha$-perylene. 
logical evolutions of perylene films grown on different substrates $\left(\mathrm{Al}_{2} \mathrm{O}_{3} / \mathrm{Si}\right.$ and $\left.\mathrm{Au} / \mathrm{glass}\right)$ and compare our findings to the results obtained in a previous study. ${ }^{7}$

\section{EXPERIMENT}

In order to study the effect of the substrate and bottom layer on the morphology and structure of the organic thin film, perylene films have been deposited on top of $\mathrm{Al}_{2} \mathrm{O}_{3} / \mathrm{Si}$ and Au/glass substrates. The glass substrates (Schott AG, D263) and Si substrates were cleaned with warm isopropanol $\left(35^{\circ} \mathrm{C}\right)$ in an ultrasonic bath and dried with nitrogen gas. Subsequently, $50 \mathrm{~nm}$ thin $\mathrm{Al}_{2} \mathrm{O}_{3}$ films were deposited on $\mathrm{Si}$ by employing reactive dc magnetron sputtering. The $50 \mathrm{~nm}$ $\mathrm{Au}$ layer was deposited on glass substrates in a thermal evaporation system ( $p \approx 10^{-5}$ Torr). Afterwards, the perylene films were prepared with different thicknesses $(50-2000 \mathrm{~nm})$ at a constant deposition rate of $2 \AA / \mathrm{s}$ under a vacuum of $10^{-6}$ Torr. The deposition rate and the thickness of the perylene films were controlled with a quartz crystal microbalance. Perylene powder (Sigma-Aldrich, 99.5\%) was used without any further purification.

$\mathrm{X}$-ray diffraction (XRD) in $\theta-2 \theta$ mode was employed to follow the evolution of the crystalline order perpendicular to the substrate plane of the perylene film. The distance between crystalline planes ( $d$ value) and the crystallinity of the films are directly determined from the XRD peak position, peak intensity, and peak width, respectively. The grain density, dislocation number density, and surface roughness of the perylene samples were determined from atomic force microscopy (AFM) images. To have more accurate statistics, the grain number is counted and averaged from six highcontrast AFM images (with a scan size of $10 \times 10 \mu \mathrm{m}^{2}$ ) for each sample. Furthermore, to characterize the lateral correlation of the surface, the radially averaged height-height correlation length has been calculated from the AFM images. This provides quantitative information about the average structure size on the surface. ${ }^{12}$

The surface free energy $\sigma$, partitioned into polar $\sigma^{p}$ and dispersive $\sigma^{d}$ contributions, was determined for samples deposited on different substrates. By measuring the contact angle with a Krüss DSA-10 contact angle goniometer, we have calculated the surface free energy by the OwensWendt-Rabel-Kälble (OWRK) method. ${ }^{13}$ This method is based on the measurement of contact angles of two or more liquids. Thereby, one obtains a set of values (contact angle $\alpha$, liquid tensions $\sigma_{l}^{d}$ and $\sigma_{l}^{p}$ ) for each liquid. This set of values is introduced into the OWRK equation:

$$
\frac{(1+\cos \alpha) \sigma_{l}}{\underbrace{\sqrt{\sigma_{l}^{d}}}_{y}}=\sqrt{\sigma_{s}^{p}} \underbrace{\sqrt{\frac{\sigma_{l}^{p}}{\sigma_{l}^{d}}}}_{x}+\underbrace{\sqrt{\sigma_{s}^{d}}}_{b} .
$$

By plotting the obtained $y$ values as a function of $x$ and performing a linear fit, the polar contribution of the surface free energy $\sigma^{p}$ can be obtained from the slope of the straight line and the dispersive contribution $\sigma^{d}$ can be obtained from the intercept. We used three different liquids for the evaluation of the contact angle, ultrapure water, diodomethane, and

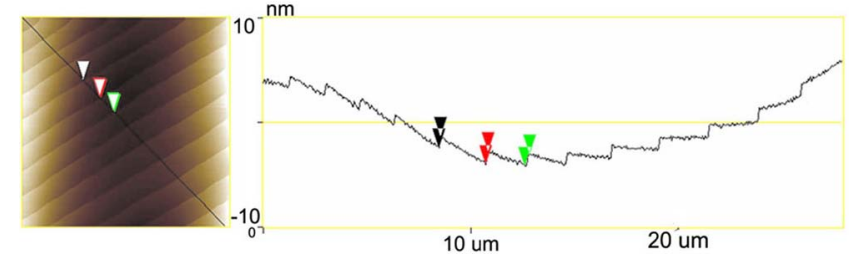

FIG. 2. (Color online) AFM image of a single crystal of perylene.

ethyleneglycol. By measuring the surface free energies of the perylene film $\left(\sigma_{\text {per }}^{d}, \sigma_{\text {per }}^{p}\right)$ and of the substrate $\left(\sigma_{s}^{p}, \sigma_{s}^{d}\right)$, the adhesion energy

$$
E_{a}=2\left(\sqrt{\sigma_{s}^{d} \sigma_{\mathrm{per}}^{d}}+\sqrt{\sigma_{s}^{p} \sigma_{\mathrm{per}}^{p}}\right)
$$

was calculated. ${ }^{14}$ In the same way, the cohesive energy has been determined:

$$
E_{c}=2\left(\sigma_{\mathrm{per}}^{d} \sigma_{\mathrm{per}}^{d}+\sigma_{\mathrm{per}}^{p} \sigma_{\mathrm{per}}^{p}\right) .
$$

\section{RESULTS AND DISCUSSION}

At first, several single crystals of perylene were prepared by sublimation. These crystals were studied to determine the crystalline structure and the growth of perylene crystals. The AFM image in Fig. 2 shows very smooth terraces and an average roughness value of $3 \mathrm{~nm}$. The vertical separation of the parallel planes, shown in Fig. 2, obtained from the AFM section analysis is $10.1 \pm 0.8 \AA$, which is in excellent agreement with the $d$ value obtained from XRD $(10.12 \pm 0.1 \AA)$. The $d$ value is the distance between two adjacent (001) crystalline planes as determined by XRD. This similarity confirms that the observed planes in the AFM image correspond to the (001) crystalline plane. The step height is close to the lattice constant of the $c$ axis in the crystalline bulk phase reported for perylene ( $c$ value), which is $10.26 \AA .{ }^{15}$ Hence in the single crystals, the molecules are ordered in the (001) direction. The small difference between the lattice constant and interlayer spacing is due to the tilt of the $c$ axis from the (001) surface normal, which amounts to around $\approx 14^{\circ}$.

\section{A. Perylene $/ \mathrm{Al}_{2} \mathrm{O}_{3} / \mathrm{Si}$}

The choice of this particular substrate has been motivated by the desire to use perylene as the active layer in OFETs. Considering this interest and the fact that a bottom gate is separated from the organic layer by an oxidic film, it is worthwhile to investigate the growth of perylene on $\mathrm{Al}_{2} \mathrm{O}_{3}$.

The morphology of perylene films deposited with different thicknesses (100-2000 nm) at a constant deposition rate of $2 \AA$ /s is presented in Figs. 3(a)-3(h). For a low film thickness $(100 \mathrm{~nm})$, the perylene grain size is around $300-600 \mathrm{~nm}$. By increasing the thickness, the surface morphology changes [see Figs. 3(b)-3(h)]. Then the substrate surface is covered with films consisting of larger grains of $500-1000 \mathrm{~nm}$ in lateral direction. For samples with a film thickness between 200 and $1000 \mathrm{~nm}$, the grain size does not considerably change with increasing film thickness. However, for the thickest film $(2000 \mathrm{~nm})$, the grains become larger $(1000-1500 \mathrm{~nm})$ and adopt a rectangular shape. 


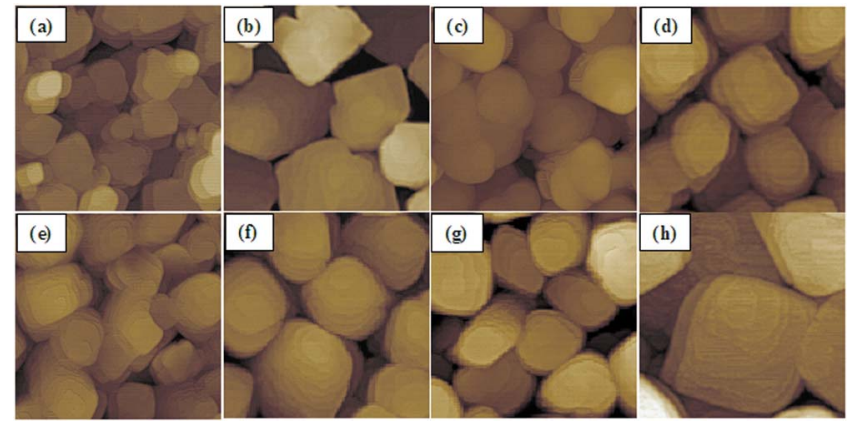

FIG. 3. (Color online) (a)-(h) show the AFM images (scan size of 2.5 $\times 2.5 \mu \mathrm{m}^{2}$ ) for perylene films with different thicknesses grown on $\mathrm{Al}_{2} \mathrm{O}_{3} / \mathrm{Si}$. The thicknesses for samples (a)-(h) are 100, 200, 300, 400, 500, 700,1000 , and $2000 \mathrm{~nm}$, respectively.

These trends can be explained as follows: The initially mobile perylene molecules move over the substrate surface and aggregate to form a high density of grains (for the $100 \mathrm{~nm}$ sample). The further increase in the grain size upon increasing the film thickness can be considered as a consequence of the coalescence of the grains. During subsequent growth, these grains start to overgrow each other. In Fig. $3(\mathrm{~h})$, this aggregation of grains is visible. In comparison with our previous films, which had been deposited on glass substrates, ${ }^{7}$ the new samples, deposited on Si substrates, have a smaller grain size. For example, the $700 \mathrm{~nm}$ thick perylene film contains grains with a size around $1500 \mathrm{~nm}$ on the glass substrate while the size was $1000 \mathrm{~nm}$ on the $\mathrm{Si}$ substrate.

For perylene $/ \mathrm{Al}_{2} \mathrm{O}_{3} / \mathrm{Si}$ samples with low thicknesses $(<50 \mathrm{~nm})$, the small grain size leads to a high grain number density. Because of grain coalescence in thicker samples, the grain size increases, which results in a decreasing number density of the grains (see Fig. 4). The formation of dislocations can be observed for film thicknesses above $100 \mathrm{~nm}$. Since each grain contains at least one screw dislocation, the dislocation number density has a value similar to the grain number density and they both decrease with thickness.

To quantify the grain size of the surface in more detail, the height-height correlation length of the film surface has been calculated (Fig. 4). For thinner films (thinner than $1000 \mathrm{~nm}$ ), the calculated correlation length is around

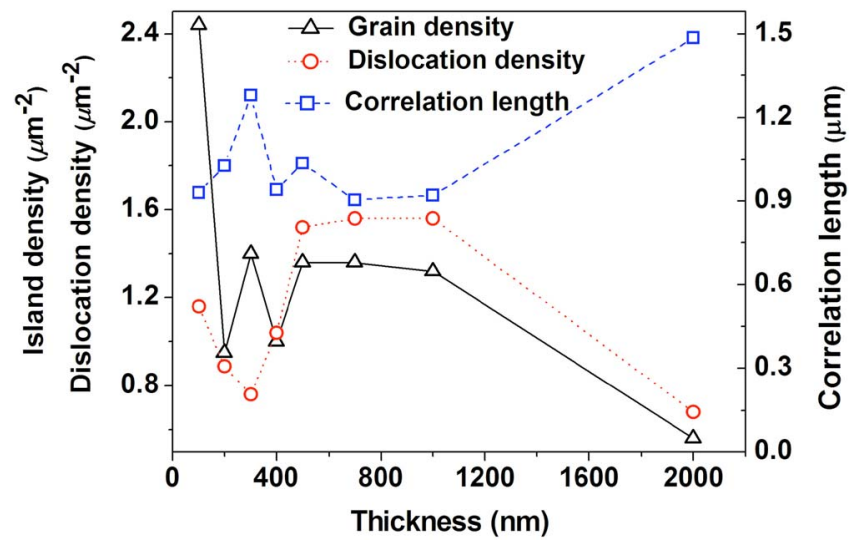

FIG. 4. (Color online) Dislocation and grain number density and correlation length obtained from AFM images for perylene on $\mathrm{Al}_{2} \mathrm{O}_{3} / \mathrm{Si}$.

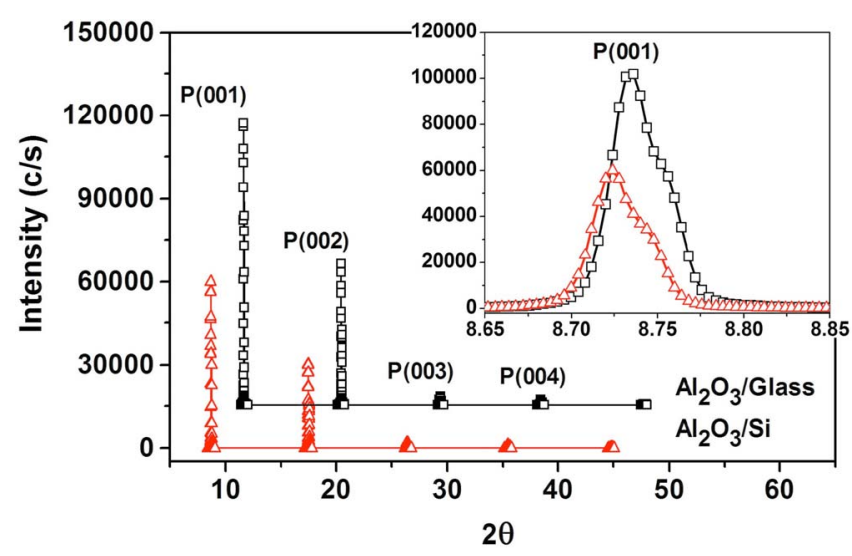

FIG. 5. (Color online) XRD $(\theta-2 \theta)$ patterns obtained for a $1000 \mathrm{~nm}$ thick perylene film deposited on $\mathrm{Si}$ and glass (the second curve is shifted by $3^{\circ}$ to the right). For detailed comparison the intensity of the (001) peak is shown in the inset.

$1000 \mathrm{~nm}$ and increases to $1500 \mathrm{~nm}$ for the highest thickness, which is in good agreement with the trend obtained for the grain size. According to the definition of the correlation length, this agreement can be explained by the fact that all grains have a mound shape. Therefore, the distance between their highest points provides an estimate of their lateral size.

The results obtained from the XRD measurements show a high crystalline quality for the samples grown on the $\mathrm{Si}$ substrate. In Fig. 5, XRD results of two different substrates have been compared. The crystalline structure shows an ordering in the (001) direction, perpendicular to the surface. However, perylene films grown on top of the glass substrate show a higher intensity and sharper peaks ${ }^{7}$ compared to the Si substrate. This finding is indicative of better crystallinity but similar crystalline structure.

To understand the effect of the substrate on the grain size and crystallinity, the drop shape analysis was carried out and values for the adhesion energy $E_{a}$ were obtained for different substrates (see Table I). The adhesion energy value obtained for $\mathrm{Al}_{2} \mathrm{O}_{3}$ /glass is lower than the adhesion energy of perylene molecules on top of $\mathrm{Al}_{2} \mathrm{O}_{3}$ on $\mathrm{Si}$. Both energies are smaller than the cohesive energy of perylene molecules. The cohesive energy is the energy gained when the perylene molecules grow on a perylene film and corresponds to $77.3 \pm 10.8 \mathrm{mN} / \mathrm{m}$. By considering these different values, the observed trends can be explained as follows: As the adhesion energy is lower than the cohesive energy, the perylene molecules prefer to stick to each other rather than to stick to the surface. Hence the maximum in binding energy is gained if the molecules grow in an upright standing arrangement, which maximizes the area contributing to the moleculemolecule interaction while at the same time minimizing the substrate-molecule interaction. Thus, the molecules grow in

TABLE I. Adhesion energy and roughness for different surfaces.

\begin{tabular}{lcc}
\hline \hline \multicolumn{1}{c}{ Surface } & $E_{a}(\mathrm{mN} / \mathrm{m})$ & Surface roughness $(\mathrm{nm})$ \\
\hline $\mathrm{Al}_{2} \mathrm{O}_{3} /$ glass & $65.2 \pm 2.3$ & 0.27 \\
$\mathrm{Al}_{2} \mathrm{O}_{3} / \mathrm{Si}$ & $69.8 \pm 2.1$ & 0.22 \\
$\mathrm{Au} /$ glass & $83.0 \pm 2.4$ & 1.3 \\
\hline \hline
\end{tabular}




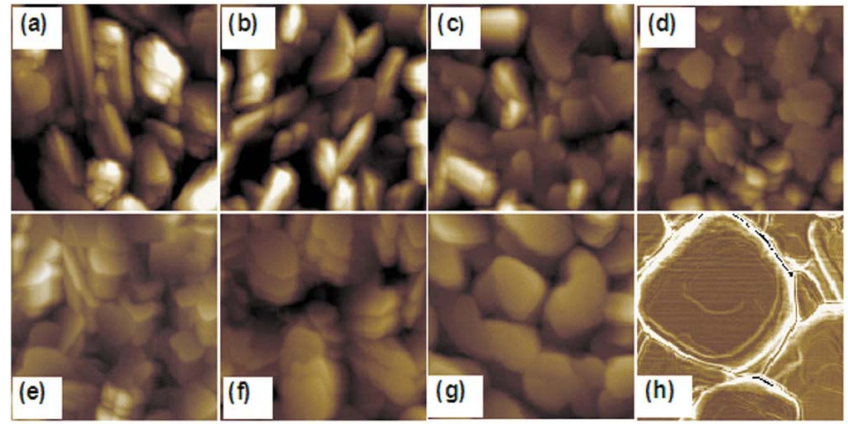

FIG. 6. (Color online) (a)-(g) show AFM images (scan size is 2.5 $\times 2.5 \mathrm{\mu m}^{2}$ ) for perylene films with different thicknesses grown on Au layer. The thicknesses for samples (a)-(f) are 50, 100, 200, 300, 400, 700, and $1000 \mathrm{~nm}$, respectively. (h) is the edge-contrast view of $(\mathrm{g})$, and the scan size is $1 \times 1 \mu \mathrm{m}^{2}$.

an almost upright standing configuration like in the bulk of perylene. Therefore, the crystalline texture in the (001) direction can be observed already for the samples with the smallest thickness.

The lower value of the adhesion energy for the case of $\mathrm{Al}_{2} \mathrm{O}_{3}$ on glass in comparison to $\mathrm{Al}_{2} \mathrm{O}_{3}$ on $\mathrm{Si}$ leads to a higher diffusivity of perylene molecules on $\mathrm{Al}_{2} \mathrm{O}_{3}$ on glass. Faster diffusion results in larger grains since the probability that a molecule reaches an existing grain and is attached to the grain increases with increasing diffusion. This is supported by comparing the grain sizes of the perylene samples deposited on $\mathrm{Al}_{2} \mathrm{O}_{3}$ on glass ${ }^{7}$ with the grain sizes of perylene samples deposited on $\mathrm{Al}_{2} \mathrm{O}_{3}$ on $\mathrm{Si}$. Perylene grains grown on $\mathrm{Al}_{2} \mathrm{O}_{3}$ on glass range in size from 500 to about $2000 \mathrm{~nm}$, while perylene grown on $\mathrm{Al}_{2} \mathrm{O}_{3}$ on $\mathrm{Si}$ forms smaller grains between 300 and $1500 \mathrm{~nm}$. The larger grain size also leads to the observed better crystallinity for perylene films on $\mathrm{Al}_{2} \mathrm{O}_{3}$ on glass.

\section{B. Perylene/Au(111)/glass}

A second set of samples has been prepared on glass substrates, coated with a $50 \mathrm{~nm}$ thin crystalline $\mathrm{Au}(111)$ layer. Figure 6 shows the morphology of the perylene films with different thicknesses grown on top of $\mathrm{Au}(111)$ layers. In the case of the thinnest sample [Fig. 6(a)], the film does not completely cover the gold surface and the grains have a nonuniform rod shape. By increasing the thickness, the grains develop from the small rodlike shape towards a rectangular and larger form. This shape transition is clearly visible for increasing film thicknesses from 200 to $300 \mathrm{~nm}$, i.e., Figs. 6(c) and 6(d). Furthermore, there is no evidence of screw dislocations for any of these samples, except for the thickest sample of $1000 \mathrm{~nm}$ [see Fig. 6(h)].

This change in the grain shape is also confirmed by the roughness analysis, which is summarized in Fig. 7. The diagram shows a gradual decrease in the roughness down to $29.3 \mathrm{~nm}$ for the sample with $300 \mathrm{~nm}$ of perylene where the $\mathrm{Au}$ substrate is completely covered. The molecular layers slowly change their growth behavior until almost all rodshape islands have disappeared. In this intermediate region,

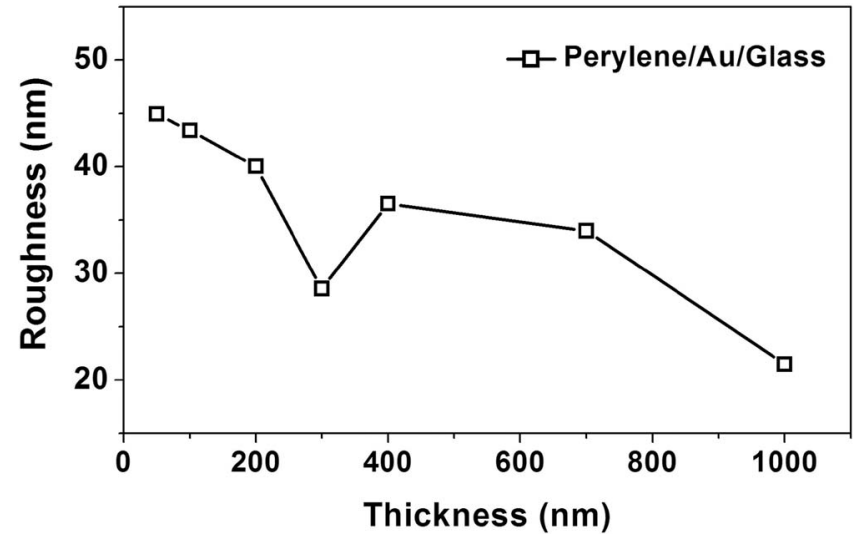

FIG. 7. Surface roughness obtained from the AFM analysis for perylene samples deposited on Au/glass with different thicknesses.

the roughness does not change and remains at around $35 \mathrm{~nm}$. For the thickest sample, the roughness slightly decreases again as the grain size increases.

In comparison, the roughness of the perylene films deposited on $\mathrm{Au}$ is significantly higher than for the perylene layers evaporated on $\mathrm{Al}_{2} \mathrm{O}_{3}$. The highest roughness was observed for films below $200 \mathrm{~nm}$ since the film is not completely closed yet and the grains grow with a high vertical growth speed. As soon as the islands coalesce, the roughness decreases since the growth shifts towards the horizontal plane and grains with a flattop are formed.

Figure 8 depicts the sequence of x-ray diffractograms obtained for different thicknesses. For the thin films $(<300 \mathrm{~nm})$, there is no evidence of a crystalline structure in the film and the only observable peak belongs to the Au film. This implies that the perylene film does not yet develop fiber texture. However, for thicker films, the molecules start to order in the (001) direction, perpendicular to the substrate and parallel to the $c$ axis. The small roughness of the bare $\mathrm{Au}$ substrate $\left(\sigma_{\text {rms }}<10 \AA\right)$ cannot be responsible for the pronounced island growth. In the light of results obtained and by considering previous studies, ${ }^{8,11}$ the different growth behaviors of perylene films on top of the different substrates can be explained by different molecule-substrate interactions. ${ }^{11}$

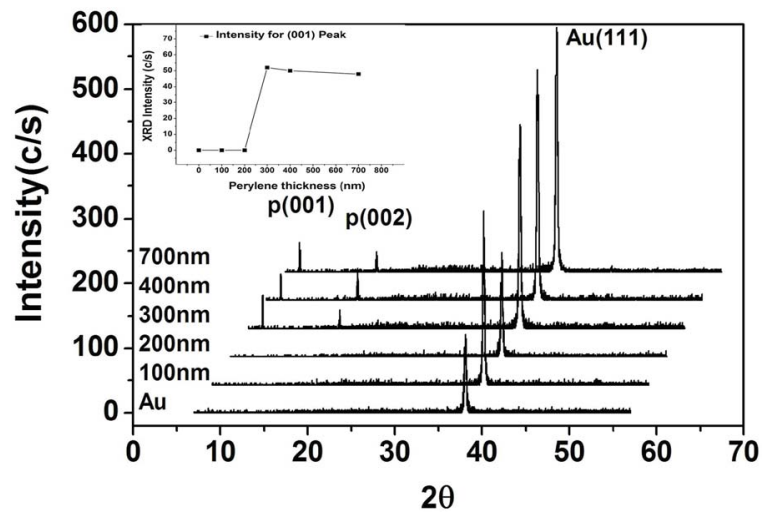

FIG. 8. (Color online) XRD pattern for perylene samples with different thicknesses grown on Au/glass. Subsequent curves are shifted by $2^{\circ}$ to the right. For ease of comparison, the intensity of the (001) peak for different perylene film thickness is shown in the inset. 
Our measurements have shown (see Table I) that the substrate-molecule interaction, i.e., the adhesion energy, for perylene on $\mathrm{Au}$ is considerably higher than the moleculemolecule interaction, i.e., the cohesive energy, which is $77.3 \mathrm{mN} / \mathrm{m}$. In this case the molecules prefer to stick to the substrate rather than to stick to each other and the adsorbtion of the molecules will occur in a lying geometry. That means the molecular plane is parallel to the substrate surface in order to maximize the binding energy to the substrate. This has been observed for numerous metallic, semiconductor, and alkali halide substrates. ${ }^{10,11,16}$ For the perylene samples deposited on the $\mathrm{Au}(111)$ layer which have a thickness above $200 \mathrm{~nm}$, the substrates are completely covered by the perylene molecules and the effect of the substrate vanishes. Subsequently, the molecules start to stand up and form a crystalline texture in the (001) direction.

\section{CONCLUSIONS}

The growth of perylene films, their morphology, and crystalline structure have been studied on two different substrates: amorphous aluminum oxide and a crystalline $\mathrm{Au}$ layer with (111) fiber texture. The grain size for both series of samples was found to increase with increasing thickness due to the molecular aggregation and overgrowth for the thicker films. Importantly, the films deposited on $\mathrm{Al}_{2} \mathrm{O}_{3}$ showed a highly textured crystalline structure and perylene molecules tend to grow in a standing geometry on this oxide substrate. On the contrary, similar samples deposited on $\mathrm{Au}(111)$ layers were characterized by a weaker crystalline order even for thick films. In this case, initially the perylene molecules prefer to arrange in the lying geometry. The different growth modes observed on these substrates can be explained in terms of the different adhesion energies of the perylene film on the substrates compared to the cohesive energy, which quantifies the molecule-molecule interaction.

${ }^{1}$ G. G. Malliaras and R. H. Friend, Phys. Today 58(5), 53 (2005).

${ }^{2}$ A. Tsumura, K. Koezuka, and T. Ando, Appl. Phys. Lett. 49, 1210 (1986).

${ }^{3}$ A. Morozov, T. Kampen, and D. Zahn, Surf. Sci. 446, 193 (2000).

${ }^{4}$ H. Peisert, T. Schwieger, J. M. Auerhammer, M. Knupfer, M. S. Golden, J. Fink, P. R. Bressler, and M. Mast, J. Appl. Phys. 90, 466 (2001).

${ }^{5}$ A. D. Carlo, F. Piacenza, A. Bolognesi, B. Stadlober, and H. Maresch, Appl. Phys. Lett. 86, 263501 (2005).

${ }^{6}$ I. Kymissis, C. D. Dimitrakopoulos, and S. Purushothaman, IEEE Trans. Electron Devices 48, 1060 (2001).

${ }^{7}$ M. Beigmohamadi, P. Niyamakom, A. Farahzadi, S. Kremers, T. Michely, and M. Wuttig, Phys. Status Solidi (RRL) 2, 1 (2008).

${ }^{8}$ S. Verlaak, S. Steudel, P. Heremans, D. Janssen, and M. S. Deleuze, Phys. Rev. B 68, 195409 (2003).

${ }^{9}$ C. Seidel, R. Ellerbrake, L. Gross, and H. Fuchs, Phys. Rev. B 64, 195418 (2001).

${ }^{10}$ M. Eremtchenko, D. Bauer, J. A. Schaefer, and F. S. Tautz, J. Mater. Res. 19, 2028 (2004)

${ }^{11}$ A. C. Durr, N. Koch, M. Kelsch, A. Ruehm, J. Ghijsen, R. L. Johnson, J. Pireaux, J. Schwartz, F. Schreiber, H. Dosch, and A. Kahn, Phys. Rev. B 68, 115428 (2003).

${ }^{12}$ A. L. Barabasi and H. E. Stanley, in Fractal Concepts in Surface Growth (Cambridge University Press, Cambridge, 1995).

${ }^{13}$ D. H. Kaelble, J. Adhes. 2, 60 (1970).

${ }^{14}$ F. M. Fowkes and W. A. Zisman, in Contact Angle, Wettability and Adhesion (American Chemical Society, Washington, DC, 1964).

${ }^{15}$ A. Camerman and J. Trotter, Proc. R. Soc. London, Ser. A 220, 311 (1953).

${ }^{16}$ A. Koma, J. Cryst. Growth 201-202, 236 (1999). 